\title{
Prevalencia de lesiones bucales en tejido blando encontradas en la Clínica de Estomatología de la Facultad de Odontología de la Universidad de los Andes. Periodo 2015-2018.
}

\author{
Prevalence of oral lesions in soft tissue found in the Clinic of Stomatology of \\ the Faculty of Dentistry of the University of los Andes. 2015-2018 period.
}

\author{
Andreina Tejada García*
}

\section{RESUMEN}

Introducción: La epidemiología sobre alteraciones en tejidos blandos bucales es limitada cuando se compara con caries, enfermedades periodontales y maloclusiones, por lo que su estudio representa un paso adelante en la odontología más allá de los dientes. Objetivo: Describir la prevalencia de las lesiones bucales en tejido blando encontradas en la Clínica de Estomatología de la Facultad de Odontología de la ULA, del 2015 al 2018, con la finalidad de proporcionar una fuente de datos actualizada, que oriente a una mejor prevención y oportuno diagnóstico. Material y métodos: Se realizó una investigación descriptiva, documental, retrospectiva y multivariable. Resultados: De 1,000 fichas clínicas estudiadas, las patologías más frecuentes fueron: lengua saburral (50.6\%), queilitis (33.4\%), traumatismo de la mucosa de los carrillos (27.6\%), várices linguales (18.3\%), anquiloglosia (13.7\%) y agrandamiento de rugas palatinas $(11.7 \%)$. La zona con mayor alteración fue la lengua (92.1\%), mientras que el paladar fue la menos afectada (29\%). De los hábitos predisponentes a la formación de lesiones, el mordisqueo de mucosa fue el más común (16.5\%). En cuanto a los factores locales asociados, una higiene oral regular/ deficiente resultó el principal (53.8\%). Conclusión: La educación del paciente sigue siendo considerada la clave para disminuir la aparición de patologías y su evolución a entidades más graves.

Palabras clave: Patología bucal, lesiones orales, lengua saburral, queilitis, hábitos bucales.

\section{ABSTRACT}

Introduction: Epidemiological studies on soft tissue alterations in the mouth are limited when compared with caries, periodontal diseases and malocclusions, so their study represents a step forward in dentistry beyond the teeth. Objective: To describe the prevalence of soft tissue oral lesions found in the Stomatology Clinic of the ULA School of Dentistry, from 2015 to 2018, in order to provide an up-to-date data source, to guide better prevention and timely diagnosis. Material and methods: A descriptive and documentary, retrospective and multivariable research was carried out. The statistical package IBM SPSS Statistics $v 23$ was applied for the analysis. Results: 1,000 clinical records studied, the most frequent pathologies were: saburral tongue (50.6\%), cheilitis (33.4\%), traumatism of the cheek mucosa (27.6\%), lingual varices (18.3\%), ankyloglossia (13.7\%) and enlargement of palatal rugas (11.7\%). The tongue was the most affected (92.1\%), while the palate was the least affected (29\%). Of the predisposing habits to the formation of lesions, mucosal nipping was the most common (16.5\%). Regarding the associated local factors, a regular / deficient oral hygiene was the main one (53.8\%). Conclusion: Patient education is still considered the key to diminish not only the appearance of pathologies but their evolution to more serious entities.

Keywords: Oral pathology, oral lesions, saburral tongue, cheilitis, oral habits.

\section{INTRODUCCIÓN}

L a cavidad oral es asiento de una amplia diversidad de entidades que involucran variaciones anatómicas y

\footnotetext{
* Profesora de la Cátedra de Patología Clínica y Terapéutica Estomatológica. Facultad de Odontología de la Universidad de los Andes. Bogotá, Colombia.

Recibido: 13 Abril 2019.

Aceptado para publicación: 18 Noviembre 2019.
}

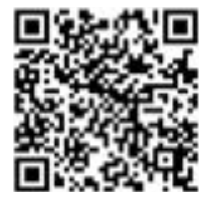

estados patológicos; continuamente se encuentra sujeta a cambios debido a diferentes factores irritantes a los que está expuesta. Las lesiones bucales en tejidos blandos pueden ser el resultado de distintos estados en la vida del ser humano, como la gestación o el envejecimiento, ser producto de disturbios emocionales, representar algunas veces las primeras manifestaciones de alteraciones sistémicas o de enfermedades infecto-contagiosas, o en el peor y más temido de los casos: el punto inicial de un cáncer oral. ${ }^{1-3}$ 
Entre las muchas clasificaciones para facilitar la identificación de las alteraciones en boca, pueden dividirse en: entidades no patológicas (también conocidas como variantes anatómicas, que engloba aquellos cambios evolutivos que sufren los tejidos durante la vida del individuo y son tan frecuentes e inocuas que no representan una patología), y entidades patológicas o lesiones bucales, que son aquellas alteraciones en la mucosa como resultado de traumatismos, infecciones bacterianas, virales o micóticas, o asociadas con padecimientos sistémicos, nutricionales, de etiología autoinmune o neoplásica. Hay patologías que se presentan en zonas específicas, como es el caso de la ránula en el piso de la boca o la estomatitis nicotínica en el paladar; sin embargo, existen otras que pueden aparecer en varios sitios de la mucosa, lo que puede llegar a dificultar su diagnóstico. ${ }^{4-7}$

Por otra parte, las lesiones bucales pueden asociarse también a un estilo de vida y hábitos poco saludables en los pacientes, que no sólo las agravan sino que también pueden originarlas; como es el caso del tabaquismo, el alcoholismo, el mordisqueo de mucosas y la masticación de chimó (tabaco curado), cuyas manifestaciones van a depender del sitio de colocación, y una higiene oral deficiente. ${ }^{8-10}$

Los conceptos de salud dental y salud oral casi siempre han sido usados como sinónimos. Sin embargo, cuando se utilizan por lo general se hace referencia a los órganos dentarios y a la higiene bucal, pasando por alto patologías de los tejidos blandos y estructuras adyacentes de la cavidad oral; la información sobre la distribución y frecuencia de lesiones de la mucosa bucal se ha reportado en diferentes países, pero suele estar restringida a muy pocas lesiones por estudio. ${ }^{11}$

En los últimos años, en Latinoamérica se han realizado investigaciones al respecto, todas ellas abordadas desde distintos puntos, como etiología, patogénesis, clínica e histopatología; no obstante, siguen siendo muy pocas en comparación con otras patologías como caries, enfermedades periodontales y maloclusiones. Estudios reportan que aproximadamente $10 \%$ de individuos mayores de 35 años tienen cuando menos una alteración en boca, y que por lo general es raro que haya sido registrada por su odontólogo, porcentaje elevado si lo asociamos con las lesiones potencialmente malignas que pudieron ignorarse. ${ }^{12}$

En Venezuela, los estudios sobre la epidemiología de estas anomalías contenidas en una misma investigación son escasos, por lo que existe un vacío de antecedentes que permitan al profesional de la salud conocer aquéllas que se evidencian con mayor frecuencia en su comunidad y preparar su accionar hacia ellas. La Facultad de Odontología de la Universidad de los Andes (FOULA), constituye el principal centro de docencia-servicio en salud oral en la ciudad de Mérida, prestando atención a pacientes, no sólo de la ciudad sino también de distintas partes del país, representando un reflejo fiel de los padecimientos de la población; y dentro de ella, la Cátedra de Patología Clínica y Terapéutica Estomatológica constituye el primer contacto de la institución con el paciente, siendo el área de especialidad en patología bucal y los responsables de la elaboración de su diagnóstico.

Por lo tanto, se planteó describir la prevalencia de lesiones bucales en tejidos blandos encontradas en los pacientes asistidos en la Clínica de Estomatología de la FOULA en el periodo 2015-2018, con la finalidad de, en primer lugar, proporcionar una fuente confiable de datos epidemiológicos actualizada, en segundo lugar orientar el plan de estudio de la materia, tomando como referencia las patologías más comunes, y en tercer lugar guiar a docentes y alumnos en la elaboración de una mejor prevención y oportuno diagnóstico.

\section{MATERIAL Y MÉTODOS}

Se realizó una investigación de tipo descriptivo, pues se estudiaron las características específicas de la población y factores asociados. Con un diseño documental, retrospectivo y multivariable, ya que la fuente de datos estuvo representada por fichas clínicas de los últimos tres años y se estudiaron varios rasgos relacionados a la patología en tejidos blandos. ${ }^{13,14}$

Para este estudio, se consideraron todas aquellas fichas clínicas durante los años 2015 a 2018 de los pacientes asistidos en el área clínica de la Cátedra de Patología Clínica y Terapéutica Estomatológica de la FOULA. Luego de haber sido sometidas a los criterios de inclusión correspondientes (datos completos, letra legible, pacientes mayores a 12 años), para una selección total de 1,000 fichas clínicas, se solicitó ante mencionada área clínica autorización para el uso de las fichas según lo contemplado en el Art. 62 y Art. 63 del Código de Deontología Odontológica Venezolano, asegurándoles que su fin sería exclusivamente académico, manteniendo el anonimato de los pacientes y demás puntos contemplados en el Art. 68 de la Ley Orgánica de Salud Venezolana. ${ }^{15,16}$

Se enumeraron las fichas clínicas y se vaciaron en el instrumento de recolección de datos elaborado especialmente para esta investigación, el cual consistió en una ficha de registro avalada previamente por un jurado de expertos en el área. El instrumento contenía primeramente el número de identificación de las fichas ordenadas cronológicamente hasta la más actual, seguido por la edad y género del paciente. Posteriormente, los hábitos 
presentados, así como los factores locales predisponentes $y$, finalmente, las lesiones encontradas con su respectiva localización. Luego de completar la recolección de datos, se procedió a totalizar lo obtenido.

El análisis de los datos fue de tipo descriptivo, lo que permitió la realización de tablas de frecuencia absoluta y gráficos en forma de barras simples y sectoriales, a través de la utilización del paquete estadístico IBM SPSS versión 23.

\section{RESULTADOS}

La población de estudio estuvo conformada por 1,000 fichas clínicas, pertenecientes al periodo 2015-2018 correspondientes a los pacientes atendidos en la Clínica de Estomatología de Facultad de Odontología de la Universidad de los Andes. La mayor afluencia de pacientes estuvo ubicada en el grupo etario de los 20 a 25 años de edad con $40.1 \%$ seguida por los de 36 a 55 años con $19 \%$. En cuanto al género, el femenino preponderó con $53.9 \%$ sobre el masculino con $46.1 \%$.

Al analizar la presencia de hábitos (Figura 1), el mordisqueo de la mucosa yugal y labial resultó el más común con $16.5 \%$, seguido del tabaquismo en $12.4 \%$, mientras que de los factores locales predisponentes a formar lesiones (Figura 2), la higiene oral regular fue el principal con 53.8\% continuando con la presencia en boca de aparatología ortodóncica en un $11 \%$.

En la lengua, $50.6 \%$ reportó patología en la forma de lengua saburral, mientras que $11.5 \%$ presentó lengua fisurada (Tabla 1).

En los labios, 33.4\% fue diagnosticado con queilitis, mientras que en $13.2 \%$ se identificó resequedad labial. Dentro de la categoría «otras lesiones» se agruparon aquéllas que se presentaron en porcentajes muy bajos (Tabla 2).

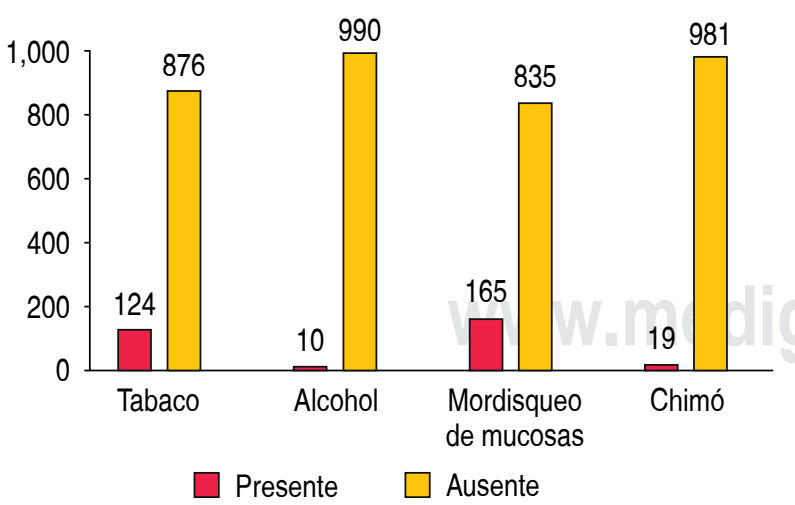

Figura 1: Hábitos de los pacientes atendidos. Modificado de Tejada, A. 2018.

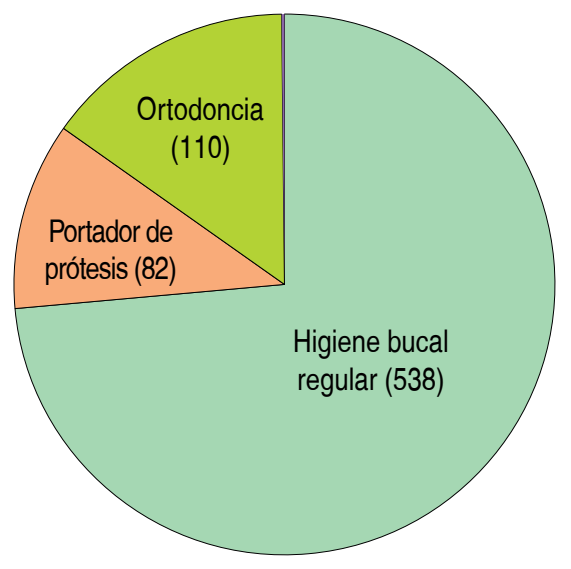

Figura 2: Factores locales asociados con lesiones en tejidos blandos. Modificada de Tejada, A. 2018.

\begin{tabular}{lr}
\multicolumn{2}{c}{ Tabla 1: Lesiones encontradas en lengua. } \\
\hline Lesión(es) & $\mathrm{n}$ \\
\hline Sin lesión & 79 \\
Lengua saburral & 506 \\
Lengua fisurada & 130 \\
Glositis migratoria benigna & 8 \\
Lengua pilosa & 109 \\
Macroglosia & 51 \\
Otras lesiones & 117 \\
Total & 1,000 \\
\hline
\end{tabular}

En la región de los carrillos, en $27.6 \%$ de las fichas clínicas se reportó traumatismo de mucosa (de tipo laceraciones y hematomas) (Tabla 3).

En el caso del paladar, el agrandamiento de rugas palatinas fue diagnosticado en $14.6 \%$ de la población de estudio, seguido de agrandamiento de la papila incisiva en $7.9 \%$ y de estomatitis subprotésica con 3.3\% (Tabla 4).

Finalmente, en la región sublingual, en $18.3 \%$ se haIlaron várices linguales y en $\mathbf{1 3 . 7 \%}$ anquiloglosia (Tabla 5).

La zona con mayor alteración fue la lengua con 92.1\%, mientras que el paladar fue la menos afectada con $29 \%$ de los pacientes.

\section{DISCUSIÓN}

Los datos epidemiológicos sobre entidades patológicas en tejido blando bucal son limitados cuando se compara 
con caries, periodontitis o maloclusiones, por lo que su estudio representa un paso adelante en la odontología interdisciplinaria (principalmente cariología y periodontología), más allá que sólo ver dientes. En la población

\section{Tabla 2: Lesiones encontradas en labios.}

\begin{tabular}{lr} 
Lesión(es) & $\mathrm{n}$ \\
\hline Sin lesión & 179 \\
Con lesión & \\
Queilitis & 334 \\
Macroqueilia & 32 \\
Microqueilia & 25 \\
Pigmentaciones melánicas & 33 \\
Traumatismo (laceraciones, hematomas) & 19 \\
Queilitis y pigmentaciones melánicas & 37 \\
Queilitis y traumatismo & 19 \\
Queilitis y macroqueilia & 23 \\
Queilitis y nevus & 15 \\
Resequedad labial & 132 \\
Nevus & 16 \\
Acné & 13 \\
Otras lesiones & 302 \\
Total & 1,000 \\
\hline
\end{tabular}

Otras lesiones: mucocele, herpes labial, afta(s), úlcera(s), fibroma, papiloma, labio fisurado.

\section{Tabla 3: Lesiones encontradas en carrillos.}

\begin{tabular}{lr} 
Lesión(es) & $\mathrm{n}$ \\
\hline Sin lesión & 655 \\
Lesión por traumatismo (laceraciones, & 276 \\
hematomas) & \\
Úlcera(s) & 4 \\
Mácula & 1 \\
Hiperplasia fibrosa & 5 \\
Agrandamiento de la glándula parótida & 3 \\
Agrandamiento del conducto de Stenon & 22 \\
Queratosis friccional & 10 \\
Pigmentaciones melánicas & 16 \\
Afta(s) & 2 \\
Leucoplasia & 2 \\
Fibroma & 4 \\
Total & 1,000 \\
\hline
\end{tabular}

\section{Tabla 4: Lesiones encontradas en paladar.}

\begin{tabular}{lr} 
Lesión(es) & $\mathrm{n}$ \\
\hline Sin lesión & 712 \\
Estomatitis subprotésica & 33 \\
Estomatitis nicotínica & 5 \\
Hiperplasia fibromatosa lateralizada & 1 \\
Úlcera(s) & 2 \\
Úvula bífida & 1 \\
Agrandamiento de la úvula & 1 \\
Agrandamiento de las rugas palatinas & 146 \\
Agrandamiento de la papila incisiva & 79 \\
Pigmentaciones melánicas & 4 \\
Afta(s) & 1 \\
Traumatismo (laceraciones, hematomas) & 10 \\
Paladar hendido/fisurado & 5 \\
Total & 1,000 \\
\hline
\end{tabular}

Tabla 5: Lesiones encontradas en región sublingual.

\begin{tabular}{lr} 
Lesión(es) & $\mathrm{n}$ \\
\hline Sin lesión en tejido blando & 614 \\
Várices linguales & 183 \\
Pigmentaciones melánicas & 3 \\
Traumatismo (laceraciones, hematomas) & 3 \\
Hiperplasia fibrosa inducida por prótesis & 1 \\
Ránula & 7 \\
Agrandamiento de carúnculas linguales & 10 \\
Afta(s) & 3 \\
Úlcera(s) & 1 \\
Anquiloglosia & 174 \\
Ausencia de frenillo & 1 \\
Total & 1,000
\end{tabular}

de 1,000 pacientes del presente estudio, la patología en lengua resultó la alteración más frecuente, siendo de tipo saburral, seguida por la queilitis y las lesiones en mucosa de carrillo por traumatismo (laceraciones y hematomas). Guzmán, Marín y Valero encontraron como principales hallazgos clínicos en una población de 400 pacientes la enfermedad en lengua, identificándose como la más común la lengua saburral, lo que coincide con los resultados de esta investigación. Entre las principales causas de la formación de la capa de saburra en la superficie 
dorsal de la lengua está la higiene deficiente; dentro de lo reflejado en el estudio, el principal factor predisponente fue precisamente una higiene oral regular o inadecuada, por lo que se comprobó su relación directa. ${ }^{17}$

Contrariamente, reportan Moret, Rivera y Cartaya que la leucoplasia y la hiperplasia fibrosa fueron las mayormente identificadas, pudiéndose asociar a la amplia diferencia temporal que hay entre ambas investigaciones, adjudicándose que actualmente se hace un énfasis especial en la educación del paciente y la prevención de patologías más severas. Coincide, sin embargo, en la frecuencia del género femenino en la segunda década de la vida como los sectores más afectados dentro de la población. ${ }^{18}$

Aldape, Padilla y Cruz también reportaron el género femenino en la segunda y tercera década de la vida como los individuos con más aparición de patologías, lo que puede deberse a que son las mujeres jóvenes las que más acuden a consulta odontológica dándole una mayor prioridad a su salud y estética. No obstante, difiere de este trabajo en que la hiperplasia fibrosa y el mucocele fueron las alteraciones en tejido blando más diagnosticadas. El sitio de mayor afección fueron los labios, lo que tiene similitud con los resultados aquí obtenidos donde la lengua y los labios son las estructuras anatómicas más susceptibles. ${ }^{19}$

En cuanto a los hábitos encontrados, la mayor parte de la literatura identifica el tabaquismo como el principal asociado a presencia de patologías en cavidad oral. Dista un poco del resultado de este trabajo, donde el mordisqueo de mucosa labial o yugal fue el más frecuente, seguido del tabaquismo, lo que se corresponde con que dentro de las lesiones encontradas de forma más común se presente la lesión por traumatismos. Este hábito está íntimamente relacionado con situaciones de estrés y ansiedad del paciente, por lo que se puede asumir que la población de estudio está sometida a niveles más altos de estos factores y que la educación sobre su manejo también debiese formar parte del tratamiento. ${ }^{20,21}$

Sumado a lo anterior, un traumatismo constante en tejidos blandos puede cambiar las características del epitelio y originar alteraciones más graves; por lo que una promoción de la salud desempeña un rol fundamental para anteponerse al desarrollo de estos hábitos. Haciendo uso de la epidemiología, se puede aumentar la eficiencia de la prevención y promoción en salud. ${ }^{1}$

Por otra parte, las estructuras anatómicas más afectadas fueron la lengua y los labios, datos a tomar en cuenta al hablar de odontología preventiva en tejidos blandos, ya que precisamente estas zonas están bien establecidas entre las localizaciones frecuentes de carcinoma en boca como lo reportan Tirado y Granados en su estudio. ${ }^{22}$

\section{CONCLUSIONES}

Fueron identificadas entre las 1,000 fichas clínicas como las lesiones con mayor prevalencia en tejido blando la lengua saburral, la queilitis y las lesiones en mucosa de carrillos por traumatismo, siendo el individuo de género femenino ubicado entre la segunda y tercera décadas de la vida el más susceptible. Las estructuras anatómicas más propensas a padecer lesiones fueron la lengua y los labios. El hábito principal presentado fue el mordisqueo de la mucosa labial y yugal, y como principal factor predisponente se registró una higiene oral regular o deficiente.

El diagnóstico realizado fue meramente clínico, se sugiere, por lo tanto, hacer corroboración con diagnósticos histopatológicos y/o imagenológicos para investigaciones futuras; teniendo en cuenta que en la medida en que se elaboren en el país más estudios de patologías bucales en tejidos blandos se tendrá una realidad más acertada, que servirá para actualizar planes de estudio en las distintas facultades de odontología y fortalecer las actividades de diagnóstico, tratamiento y prevención en el ejercicio profesional.

\section{AGRADECIMIENTOS}

A la Facultad de Odontología de la Universidad de los Andes, Mérida-Venezuela y a su Cátedra de Patología Clínica y Terapéutica Estomatológica por su invaluable colaboración.

\section{BIBLIOGRAFÍA}

1. Donahue A, Leyva ER, Ponce S, Aldape B, Quezada D, Borges A et al. Distribución de condiciones y lesiones de la mucosa bucal en pacientes adultos mexicanos. Rev Cubana Estomatol. 2007; 43 (1).

2. Pacho-Saavedra JA, Piñol-Jiménez FN. Lesiones bucales relacionadas con las enfermedades digestivas. Rev Cubana Estomatol. 2006; 43 (3).

3. Brito N, González M, Bagur M. Lesiones en la mucosa bucal que se manifiestan durante el tratamiento ortodóntico. ODOUS Científica. 2007; 8 (2): 39-45.

4. Bermúdez J. Lesiones en la mucosa bucal relacionadas con el consumo del tabaco de mascar (chimó). Reporte de un caso. Ciencia Odontológica. 2011; 8 (2): 141-150.

5. García E, Anaya G, Godoy L. Manual para la detección de alteraciones de la mucosa bucal potencialmente malignas. Diario Oficial de la Federación el 23 de julio de 2003.

6. Aguas $\mathrm{S}$, Lanfranchi $\mathrm{H}$. Lesiones premalignas o cancerizables en cavidad oral. Revista de la Facultad de Odontología (UBA). 2004; 19 (47): 21-30.

7. Casian A, Trejo P, De León C, Carmona D. Hiperplasia fibrosa: reporte de un caso. Rev Clin Periodoncia Implantol Rehabil Oral. 2011; 4 (2): 74-79. 
8. Jiménez C, Ramírez R, Ortiz V, Virgüez Y, Benítez A. Identificación de lesiones en los tejidos blandos de la cavidad bucal en adolescentes con privacion de libertad en el odontopediátrico de Carapa. Acta odontológica Venezolana. 2006; 47 (2): 327-333.

9. Gonzáles-Gonzáles R, Bolgna-Molina R, Névarez-Rascón A, GilCarreón R. Lesiones frecuentes en la mucosa bucal en niños y adolescentes: revisión literaria. Rev ADM. 2011; 67 (1): 17-24.

10. Corona-Carpio M, Ramón-Jiménez R, Urgelléz-Matos W. Lesiones de la mucosa bucal en adultos mayores con prótesis dentales totales. MEDISAN. 2017; 21 (7): 813-818.

11. Axéll T, Zain RB, Siwamogstham P, Tantiniran D, Thampipit J. Prevalence of oral soft tissue lesions in out-patients at two Malaysian and Thai dental schools. Community Dent Oral Epidemiol. 1990; 18 (2): 95-99.

12. Bouquot JE. Common oral lesions found during a mass screening examination. JADA. 1986; 112: 50-57.

13. Hurtado J. El proyecto de investigación: comprensión holística de la investigación. 6a edición. Caracas, Venezuela: Ediciones Quirón, Editorial Sypal; 2010. 168 pág. 13.

14. Díaz-Narváez V, Calzadilla-Núñez A. Artículos científicos, tipos de investigación y productividad científica en las Ciencias de la Salud. Revista Ciencias de la Salud. 2016; 14 (1): 115-121.

15. Código de Deontología Odontológica. XXXIX Convención Nacional del Colegio de Odontólogos de Venezuela: San Felipe - Estado Yaracuy. Agosto de 1992.

16. Ley Orgánica de Salud. Gaceta Oficial № 36.579 de fecha 11 de noviembre 1998.

17. Guzmán-Marín B, Calero JA. Principales hallazgos clínicos en la lengua en una población de Cali. Rev Estomat. 2005; 13 (1): 5-12.
18. Moret $\mathrm{Y}$, Rivera H, Cartaya M. Prevalencia de lesiones en la mucosa bucal de pacientes diagnosticados en el Laboratorio Central de Histopatología Bucal "Dr. Pedro Tinoco" de la Facultad de Odontología de la Universidad Central de Venezuela durante el periodo 1968-1987. Acta Odontol Venez. 2007; 45 (2): 1-7.

19. Aldape B, Padilla G, Cruz B. Frecuencia de lesiones bucales histopatológicas en un laboratorio de patología bucal. Rev ADM. 2007; 64 (2): 61-67.

20. Gonzáles R, Herrera B, López I, Osorio M, Madrazo D. Principales lesiones bucales y factores de riesgo presentes en población mayor de 60 años. Rev Cubana Estomatol. 2010; 47 (1): 105-114.

21. Quintana JC, Fernández M, Laborde R. Factores de riesgo de lesiones premalignas y malignas bucales. Rev Cubana Estomatol. 2004; 41 (3).

22. Tirado L, Granados M. Epidemiología y etiología del cáncer de la cabeza y el cuello. Cancerología. 2007; 2: 9-17.

23. Mascaró, JM. Diccionario terminológico de ciencias médicas, 1977 Barcelona, Salvat Editores.

Correspondencia:

Andreina Tejada García

Urb. Belensate, calle 8, \#47 Caruyara.

República Bolivariana de Venezuela

Tel: + 582742663808 / 4147790220

E-mail: od.andreinatejada@gmail.com 